\title{
Competencias en tecnologías de información y comunicacion en estudiantes de preparatorias públicas del
} Estado de Michoacán.

Skills and communication technologies information in students preparatory of public schools State of Michoacan.

Habilidades tecnológicas em estudantes de informação e comunicação de alta estado público de Michoacan.

Joanna Koral Chávez López Universidad Michoacana de San Nicolás de Hidalgo, México Joanna.koral.ch@gmail.com

Marisol Morales Rodríguez

Universidad Michoacana de San Nicolás de Hidalgo, México marisolmoralesrodriguez@gmail.com

\section{Resumen}

El objetivo del presente estudio fue identificar las competencias tecnológicas con las que los estudiantes de bachillerato cuentan para afrontar las necesidades de su nivel educativo y determinar si existe relación entre estas.

Se basa en un enfoque cuantitativo, participaron 3000 estudiantes de nivel bachillerato de las cinco preparatorias de la UMSNH del Estado de Michoacán, se utilizó el Cuestionario de Competencias Tecnológicas del estudiante (Cabero y Llorente, 2009) que evalúa Aspectos técnicos, pedagógicos, sociales, éticos y legales, de gestión y organización escolar y Aspectos de comunicación con nuevas herramientas de comunicación. Dicho cuestionario se aplicó durante el ciclo escolar febrero -agosto 2016.

En los hallazgos se puede observar que los adolescentes cuentan con competencias tecnológicas con niveles entre buenos y regulares, en los Aspectos técnicos, pedagógicos, de gestión y organización escolar y aspectos sociales, éticos y legales así como un nivel regular en el Aspectos de comunicación con nuevas herramientas de comunicación. 
Palabras clave: Competencias tecnológicas, Estudiantes, Bachillerato

\section{Abstract}

The objective of the present study was to identify the technological skills that high school students have to meet the needs of your educational level and determine if there is a relationship between these.

It is based on a quantitative approach, the sample was 3000 students of psychology of the UMSNH of the State of Michoacán, Technological Skills Questionnaire was used student (Cabero y Llorente, 2009) evaluating technical, educational, social, ethical and legal, managerial and organizational aspects of school and communication with new communication tools aspects. Such questionnaire applied during February -August 2016 school year.

The results it can be seen that teenagers have technological skills with levels between poor, fair and good in the technical, educational, social, management and school organization and a good level in the aspects of communication with new communication tools.

Key words: Technological Skills, Students, Preparatory.

\section{Resumo}

O objetivo deste estudo foi identificar as competências tecnológicas com que estudantes do ensino médio têm de atender às necessidades de seu nível educacional e determinar se existe uma relação entre eles.

Baseia-se em uma abordagem quantitativa, envolvendo 3.000 alunos do ensino médio nível preparatório cinco UMSNH do Estado de Michoacan, Questionário habilidades tecnológicas do aluno (Cabero e Llorente, 2009), que avalia técnica, foi utilizado aspectos educacionais, sociais, ética e legal, gestão e organização escolar e os aspectos de comunicação com novas ferramentas de comunicação. O questionário foi aplicado durante fevereiro-agosto 2016 ano escolar.

Nos resultados pode ser visto que os adolescentes têm níveis tecnológicos de competências entre boa e regular, em técnica, educacional, gestão e organização escolar e aspectos sociais, éticos e legais, bem como um nível regular nos aspectos da comunicação com novas ferramentas de comunicação. 
Palavras-chave: competências tecnológicas, estudantes, segundo grau.

Fecha recepción: Enero $2016 \quad$ Fecha aceptación: Junio 2016

\section{Introducción}

En esta época de la tecnología es importante la adquisición de competencias tecnológicas, para una buena calidad de aprendizaje y conocimientos, este punto se manifiesta en el proceso de enseñanza a los alumnos teniendo como objetivo integrar y fomentar en los procesos de enseñanza y aprendizaje el uso de las Tecnologías de la Información y Comunicación (TIC) en todas las instituciones, puesto que para generar un mayor conocimiento las competencias tecnológicas son primordiales.

Por ello primeramente es muy relevante dejar en claro lo que bien se cuestiona Acuña (2012) ¿Quién es un Nativo Digital?, si bien menciona, son los sujetos que nacieron y crecieron con las nuevas tecnologías, mismas que conforman el entorno tanto social como familiar, es decir, los que nacieron a partir de 1980, cuando comenzaba la comercialización digital. A su vez, son personas que tienen un componente psico-cognitivo diferente, es decir, tienen otra forma de pensar, resolver los problemas desde los más simples hasta los más complejos. Del mismo modo Guerra, M.A, Moreno, I. y Marquina, R. (2008) hacen mención a un conjunto de habilidades y características de los Nativos Digitales tales como: recoger información de manera rápida, disfrutar los procesos multitareas y en paralelo, les gusta los gráficos más que el texto y trabaja mejor cuando realizan tareas en red, etc.

Por otro lado están los Inmigrantes Digitales como lo define Mark Prensky Citado en Guerra, M.A et al (2008) quienes son los demás mortales que nacieron antes que los Nativos Digitales, es decir, en la era pre-digital por lo que su componente cognitivo es distinto al que utilizan los nativos. Del mismo modo, Guerra, M.A, et al (2008) mencionan un conjunto de habilidades y características de los Nativos Digitales tales como: realizan un análisis deductivo, hacen estructuras mentales definidas por los procesos paso a paso, siguen instrucciones y solo resuelven un problema a la vez y su aprendizaje está basado en 
el vínculo con conocimientos previos. De tal forma que los inmigrantes realizan tareas de manera normal y frecuente, como cuando envían un correo e informan a la persona que ya lo hizo y con ello corroborar que le llego, para corregir un documento hay que imprimirlo y corregirlo y cuando adquiere algo nuevo hay que leer el instructivo, es decir la manera en la que analizan la información es distinta entre los nativos e inmigrantes digitales.

Es por ello que resulta importante, hacer énfasis que la información que se consulta u obtiene a través de Internet deba ser monitoreada, ya que mucha puede ser información no científica, no verídica y que sirve en ocasiones como medio para encontrar pornográfica, en consecuencia la información refleja el abismo que existen entre maestros, padres y alumnos.

\section{El significado de las Competencias}

Como lo indica Guadarrama (2012) precisar una definición del término competencia es una actividad muy compleja pero necesaria, ya que dicho concepto ha sido muy sonado y poco reflexionado, puesto que existe una estrecha relación con disciplinas como la Psicología, la Pedagogía, la Sociología, las Ciencias Políticas, la Educación, las Ciencias Antropológicas y las disciplinas administrativas y laborales.

En consecuencia es de suma importancia conceptualizar el término Competencia en un primer sentido como Corominas, 1998 citado en Monereo (2003) lo define, dicho término deriva de la palabra latina competere, cuya raíz viene de dos verbos en castellano: "competer" que significa "ir una cosa al encuentro de otra, encontrarse, coincidir, pertenecer" y "competir" que se asimila a ser "adecuado o apto" para una determinada actividad, es decir, ser competitivo en una tarea es una resolución de la misma de manera eficaz. En esta misma línea de pensamiento Guadarrama (2012) en la figura 1 realiza un análisis polisémico del término competencia que permite aclararlo un poco, aunque también surgen tres términos como competitividad, incumbencia y capacidad, que participaron en la confusión del mismo y cuyos significados se muestran en la tabla 1. Así mismo menciona que la competencia "expresa la capacidad de actuar en más de un campo y, mapas que conocimientos, conlleva de manera natural su uso eficaz" 
Figura 1 Expresión de significados lexicológicos

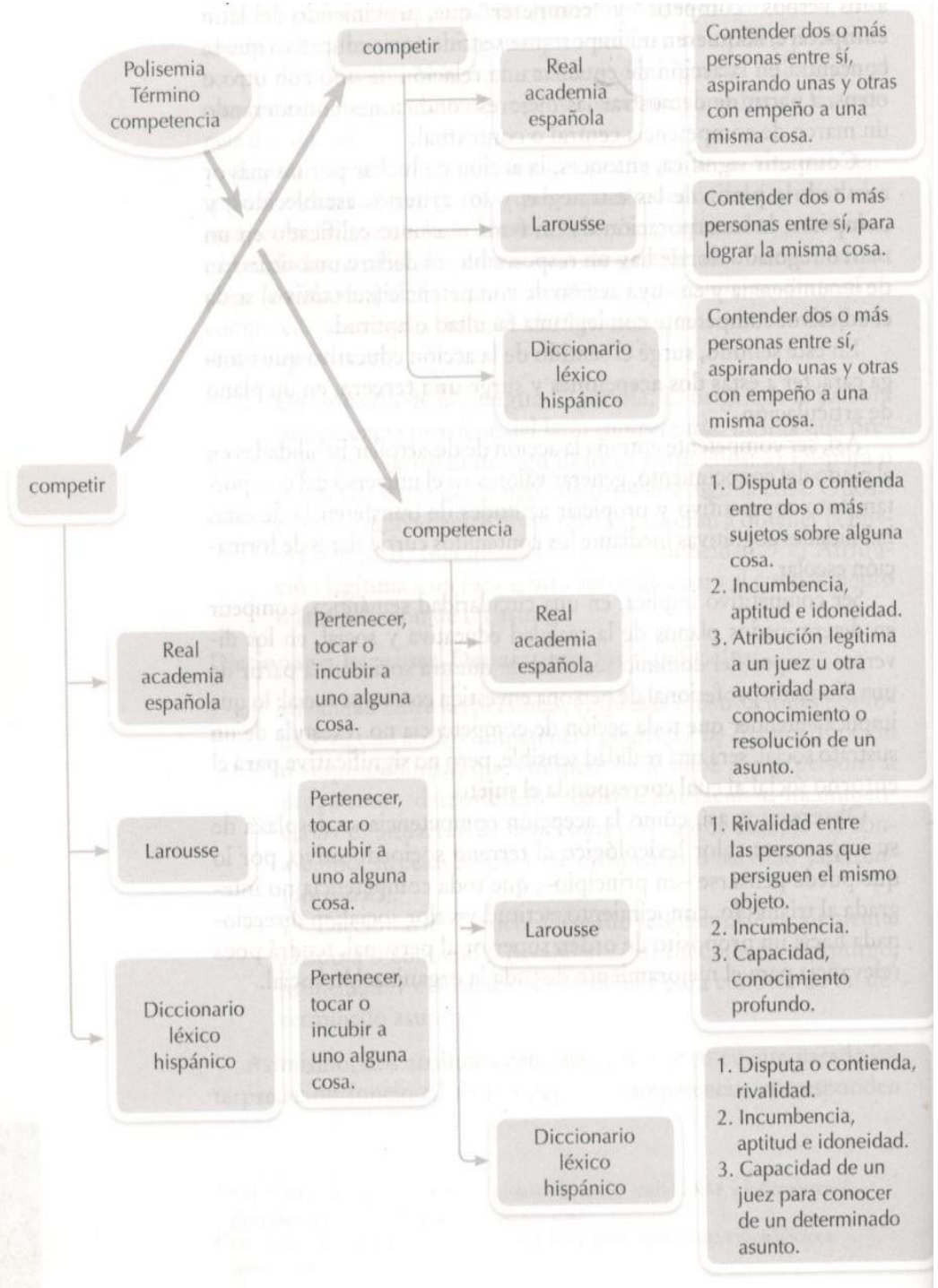

Fuente: Guadarrama (2012)

Tabla 1 Términos asociados a Competencia

\begin{tabular}{|c|c|}
\hline \multicolumn{2}{|r|}{ Competencia } \\
\hline Acepción & Significado \\
\hline Competitividad & Competir con otros para ser el mejor \\
\hline Incumbencia & Ámbito de competencia \\
\hline Capacidad & $\begin{array}{l}\text { Saberes Básicos: } \\
\text { Hacer algo-conocimientos base } \\
\text { Saber Cómo-habilidades en bases conocimiento }\end{array}$ \\
\hline
\end{tabular}

Fuente: Guadarrama (2012) 
También es importante entender la diferencia que Monereo (2003) realiza entre estrategia y competencia, siendo la primera una acción específica para resolver un tipo contextualizado de problemas y la segunda el dominio de una amplia colección de estrategias en un determinado ámbito o escenario de la actividad humana, en este sentido también es importante entender el significado de ser eficaz, término que puede ser medido con tres requisitos, la distancia entre el resultado buscado y el logrado, la calidad del proceso de resolución y por último el control y regulación consciente sobre el proceso y el resultado obtenido.

Otra explicación que Pimienta (2012) realiza al término competencia es el estar apto para alguna actividad, es decir, "el desempeño o la actuación integral del sujeto, lo que implica conocimientos factuales o declarativos, habilidades, destrezas, actitudes y valores, dentro de un contexto ético, aunque no todo desempeño es una competencia, pero una competencia no puede prescindir de un desempeño puesto que se hace evidente por medio de este último".(p.2)

Con la Figura 2, Pimienta (2012) indica como la competencia surge de la intersección entre los conocimientos factuales y declarativos (saber conocer), habilidades y destrezas (saber hacer) y actitudes y valores (saber ser), pensando en ello, nos es conveniente asegurar que una competencia es un conjunto de conocimientos, habilidades, destrezas, procedimientos, actitudes y valores, ya que durante el desempeño, esencialmente todo eso es lo que activa una competencia en un sujeto.

Figura 2. Competencia y sus saberes

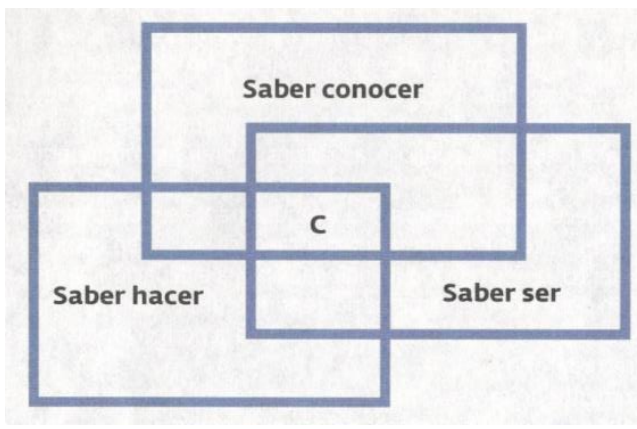

Fuente: Pimienta (2012) 
Ahora es importante referirse a la tabla que Guadarrama (2012) muestra en su libro Competencias docentes, respecto a la diversidad de definiciones del término competencia como se observa en la tabla 2.

Tabla 2. Definiciones del término competencia.

\begin{tabular}{l} 
Definiciones del término competencia \\
\hline "Sistema más o menos especializado de capacidades y destrezas que son necesarias o suficientes \\
para alcanzar un objetivo específico" (F.E. Weinert, 2001) \\
"Capacidad para responder a las demandas complejas y llevar a cabo tareas de forma adecuada. \\
Supone una combinación de habilidades prácticas, conocimientos, motivación, valores éticos, \\
actitudes, emociones y otros componentes sociales y de comportamiento que se movilizan \\
conjuntamente para lograr una acción eficaz" (OCDE/DeSeCo,2002) \\
"Combinación de atributos-respecto al conocimiento y sus aplicaciones, aptitudes, destrezas y \\
responsabilidades- que describen el nivel o grado de suficiencia con que una persona es capaza \\
de desempeñarla" (Proyecto Tuning,2003) \\
"Capacidad subyacente en una persona que está causalmente relacionada con el desempeño, \\
referido a un criterio superior o efectivo, en un trabajo o una situación" (Spencer y Spencer, \\
1993) \\
"Una competencia básica es un conjunto de conocimientos, actitudes, habilidades y destrezas que \\
permiten a un individuo responder a las demandas de una situación concreta. No se trata de un \\
concepto meramente prágmatico sino que tiene un contenido ético, porque se considera \\
competente al individuo que es capaz de desempeñar adecuadamente una tarea valiosa para sí \\
mismo y para la sociedad” (J.A.Marina, 2007) \\
“ Competencia es la habilidad para afrontar demandas externas o desarrollar actividades y \\
proyectos de manera satisfactoria en contextos complejos, implica dimensiones cognitivas y no \\
cognitivas: conocimientos, habilidades cognitivas, habilidades prácticas, actitudes, valores y \\
emociones" (A.I. Pérez Gómez, 2007)
\end{tabular}

Fuente: Guadarrama (2012)

Otros conceptos que son necesarios definir como bien lo hace Monereo (2003) son los términos capacidad la cual es innata ya que es una disponibilidad génetica del organismo, por lo que no requiere aprendizaje, por otro lado las estrategias y competencias las cuales se aprenden con la ayuda o enseñanza de otros seres humanos en comunidades de práctica, en las cuales se va adquiriendo dominio, ya que dichos términos implican una colección de 
acciones aprendidas, autorreguladas, contextualizadas y de dominio variable, de igual manera las técnicas y habilidades mismas que si son aprendidas, enfatizando que la habilidad es el resultado de una capacidad que se ha desarrollado con la ayuda de una técnica especifica.

\section{Clasificación de competencias}

Hoy en día, muchas universidades han realizado reformas educativas en sus planes de estudio, con la finalidad de poder desarrollar ciudadanos capaces de resolver problemas y colaborar para y con la sociedad, con la intensión de poder visualizar los nuevos requerimientos que la sociedad va presentando. En este sentido organismo europeos integraron las recomendaciones del proyecto Tuning como lo menciona González y Wagenaar,2003; citado en Blanco (2009), el cual refiere de una formación superior basada en competencias haciendo alusión manifiesta a la formación en competencias generales, con la finalidad de asegurar la empleabilidad de los profesionistas nuevos, entendiendo el término empleabilidad como:

...la capacidad y seguridad a la hora de retener un empleo, de aumentar su productividad y ser competitivo y poder decidir y moverse dentro de las variadas opciones ocupacionales que pueden plantearse en su entorno laboral. Depende de la capacidad de "aprender a aprender" para adaptarse a cambios y nuevas oportunidades en un entorno muy cambiante. (p.20)

Con lo anterior en el ámbito académico el proyecto Tuning propone competencias específicas las cuales se vinculan con las áreas temáticas, ya que están relacionadas con el saber específico del área, por otro lado las Competencias generales: que vienen a ser los rasgos compartidos que pueden producirse en cualquier situación. Y en el contexto universitario el proyecto emplea Competencias instrumentales: refiriéndose a la capacidad de comprender y emplear pensamientos e ideas, ser capaz de regular el tiempo y las estrategias para el aprendizaje, así como la resolución de problemas y la toma de decisiones, a su vez contar con destrezas tecnológicas (manejo de maquinaria como computadoras) y lingüísticas (comunicación oral y escrita así como conocer una segunda lengua), también son necesarias las Competencias interpersonales: alude a la destreza social, trabajar en equipo con compromiso ético y social, saber expresar sentimientos, ser 
crítico y autocritico con el debido respeto hacia los demás, por último las Competencias sistémicas: se refiere a la habilidad de planificar cambios con la intensión de mejorar los sistemas y a su vez plantear nuevos sistemas. Esta competencia integradora necesita de la obtención de las instrumentales e interpersonales (González y Wagenaar, 2003; citado en Blanco, 2009).

Fallows y Steven, 2000; citado en Blanco (2009) realizaron una revisión respecto a las experiencias universitarias, en las cuales encontraron la necesidad de desarrollar cinco competencias en las que coincidieron la mayoría de las universidades, siendo estas:

- Habilidades para la utilización de nuevas tecnologías.

- Habilidades de comunicación general.

- Trabajo en equipo, ética, reconocimiento de la diversidad.

- Competencias personales como "gestión del tiempo", "responsabilidad", "planificación".

- Gestión de la información: búsqueda, selección, análisis y evaluación de la información procedente de diversas fuentes.

Estas competencias generales a desarrollar por el alumno aunque surgen del acuerdo de un gran número de universidades, no son una propuesta única ya que también se ve mezclada por la postura histórica y filosófica de las instituciones (Blanco, 2009).

Guadarrama (2012) alude que es importante plantear que las competencias existen por la necesidad de resolver problemas y situaciones, siendo estas muy necesaria para dicha actividad o para las situaciones que solicita la sociedad al compartir el planeta que habitamos y que debemos cuidar, con el objetivo de lograr un bien colectivo. En consecuencia, el Proyecto Definición y Selección de competencias que comenta Guadarrama (2012) clasificó las competencias en tres categorías y a su vez en tres subcategorías:

Categoría 1. Usar herramientas de manera interactiva.

- Habilidad para el uso interactivo del lenguaje, los símbolos y los textos.

- Capacidad para el uso interactivo del conocimiento y la información.

- Habilidad para el uso interactivo de la tecnología. 
Categoría 2. Interactuar en grupos heterogéneos.

- Habilidad para relacionarse bien con otros.

- Habilidad para cooperar y trabajar en equipo.

- Habilidad para administrar, gestionar y resolver conflictos.

Categoría 3. Actuar de manera autónoma.

- Habilidad de actuar dentro del gran esquema.

- Habilidad de formar y conducir planes de vida y proyectos personales.

- Habilidad de afirmar derechos, interés, límites y necesidades.

En conclusión Guadarrama (2012) apunta que la competencia es una condición que permite conducirse eficazmente ante las situaciones de la vida diaria y para lo cual no solo es indispensable el conocimiento sino además resolver que hacer con él y para qué es, ya que se integran habilidades y actitudes o valores desarrolladas y que se ven reflejadas en el desempeño, creando evidencias que pueden ser transferibles a distintos contextos.

Por otra parte Monereo (2003) realiza una clasificación de las competencias denominándolas socio-cognitivas básicas, las cuales se consideran necesarias para desarrollarse en la sociedad del conocimiento. Estas competencias están agrupadas en cuatro bloques; en el primer bloque denominado Competencias para buscar información y aprender a aprender: el cual se refiere al conjunto de estrategias que permiten al alumno aprender a partir de sus propios recursos, supone un aprendizaje permanente, autónomo, autorregulado, de situaciones de enseñanza no formales y estratégico. En un segundo bloque se refiere a las Competencias para aprender a comunicarse: en el cual se habla de un conjunto de estrategias que benefician el diálogo eficaz y comprensivo con la ayuda de un dispositivo, supone una comunicación con un lenguaje particular para cada disciplina, emplear distintos medios de manera paralela y priorizar los aspectos semánticos de la comunicación. El bloque tres de competencias para aprender a colaborar: se enfoca en el conjunto de estrategias que facilitan el trabajo en equipo, es decir, aprender de forma cooperativa, aprende en red y desarrollar instituciones que aprendan. Y por último las Competencias para aprender a participar en la vida pública: encaminadas al conjunto de estrategias que transforman al sujeto en un miembro activo, participativo y responsable, el 
cual supone una participación en la construcción de la identidad personal y de unas metas vitales, participar activamente en la vida pública, fomentar una actitud empática y tolerante y desarrollar una visión crítica (figura 3).

Figura 3 Competencias socio-cognitivas básicas

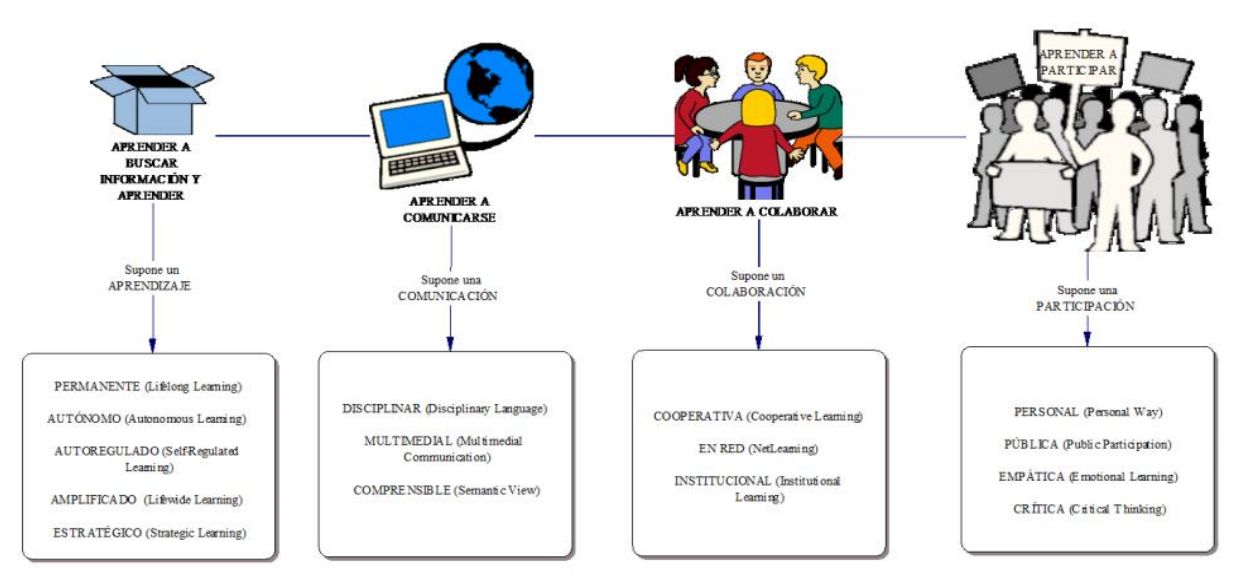

Fuente: Monereo (2003)

En cuanto a las definiciones de competencias antes mencionadas, se muestran una serie de habilidades para formar a un alumno competente que pueda sustentar sus necesidades en el campo tecnológico de información y comunicación, por ello Llorente y Cerro (2005) mencionan seis categorías, criterios o estándares formativos:

1. Operaciones y conceptos básicos en esta categoría se refiere a que los alumnos entienden perfectamente la función y anatomía de los artefactos tecnológicos (PC, software, hardware, etc.) y dominan estas funciones perfectamente.

2. Problemas sociales, éticos y humanos, es decir, que los alumnos entienden los problemas que se generan en relación con la tecnología, los aspectos éticos, culturales y humanos y un manejo adecuado y responsable en el uso de la tecnología, para que los alumnos desarrollen una actitud positiva permitiendo la optimización de su aprendizaje.

3. Herramientas tecnológicas para la productividad, en este criterio los alumnos utilizan estas herramientas para optimizar, mejorar su conocimiento, creatividad e inclusive para la creación o el mejoramiento de las tecnologías 
4. Herramientas tecnológicas para la comunicación, los alumnos utilizan las redes sociales y sitios web para la utilización y producción de información en diferentes formatos.

5. Herramientas tecnológicas para la investigación, los alumnos utilizan la tecnología con fines de investigación, reproducción y análisis de resultados para trabajos, tareas y proyectos de investigación.

6. Herramientas tecnológicas para la solución de problemas y la toma de decisiones, los alumnos manejan estas herramientas para la solución de problemas reales.

Estos criterios son los que manifiestan las competencias tecnológicas que deberían poseer los alumnos para su formación. Es por ello el objetivo de esta investigación el cual es conocer las competencias tecnológicas con los que cuentan los estudiantes de preparatorias públicas del Estado de Michoacán.

\section{MÉTODO}

El presente estudio se ciñe en una metodología cuantitativa de diseño no experimental de alcance descriptivo-correlacional.

Participantes. La muestra de estudio estuvo conformada por 3000 adolescentes estudiantes de nivel medio superior de la UMSNH. El 47.4\% son hombres y el 52.3\% mujeres. La edad promedio es de 16.8 años. El $77.9 \%$ indican tener computadora en casa y el $22.1 \%$ no disponen de una computadora como tal, confirmando con esto que cada vez son más los mexicanos que cuentan con una computadora en casa, en lo que respecta a si tienen el servicio de Internet en casa el $79.7 \%$ si tienen y el $20.3 \%$ no cuentan con el servicio.

También mencionan que el $76.6 \%$ realizan trabajos académicos en casa, el $7.4 \%$ en el centro de cómputo de la escuela y el $14.7 \%$ en el ciber ya sea cerca de su casa o cerca de la escuela. En lo que respecta a la variable sociodemográfica si utiliza el centro de cómputo de su escuela el $39 \%$ menciona si utilizarlo pero unos comentan que solo si tienen tiempo libre para trabajar sus tareas con la finalidad de avanzar y en otros casos porque no cuentan con una computadora en casa y el $60.9 \%$ no utilizan el aula de computo de la escuela ya que cuentan con todo en casa para realizar la tarea. 
Instrumentos. Fue utilizado el Cuestionario de Competencias Tecnológicas del estudiante (Llorente y Cabero, 2009). Dicho cuestionario es de tipo Likert, compuesta por 50 ítems, cuyas opciones de respuesta es de 7 puntos organizados en seis aspectos: técnicos, pedagógicos, sociales, éticos y legales, de gestión y organización escolar y Aspectos de comunicación con nuevas herramientas de comunicación. Las puntuaciones pueden oscilar entre 0 y 312 puntos, indicando una competencia las puntuaciones más altas. El instrumento tiene un índice de confiabilidad de alpha de Cronbach de .937 y por el método de dos mitades de .945 , mostrando una varianza total explicada de $48.8 \%$

Procedimiento. Para llevar a cabo el proceso, el cuestionario fue aplicado a los adolescentes al inicio del ciclo escolar febrero -agosto 2016. La aplicación fue de manera colectiva dentro del aula de clases. Para el análisis de los datos se emplearon las pruebas estadísticas chi-cuadrada y Coeficiente de correlación de Pearson.

\section{RESULTADOS}

Los hallazgos refieren, que el nivel en el Aspecto Técnico en que los jóvenes se ubican de manera predominantes es el nivel bueno, seguido del nivel regular como se puede ver en la figura 4.

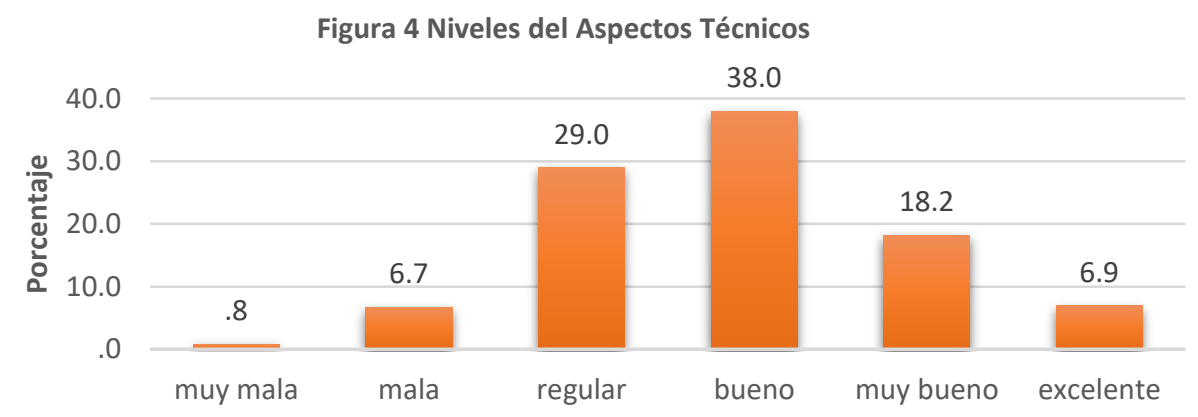

Fuente: Elaboración propia

De igual forma, se reportan en el Aspecto Pedagógico de manera preponderante el nivel regular, acompañado del nivel bueno, como se puede ver en la figura 5. 


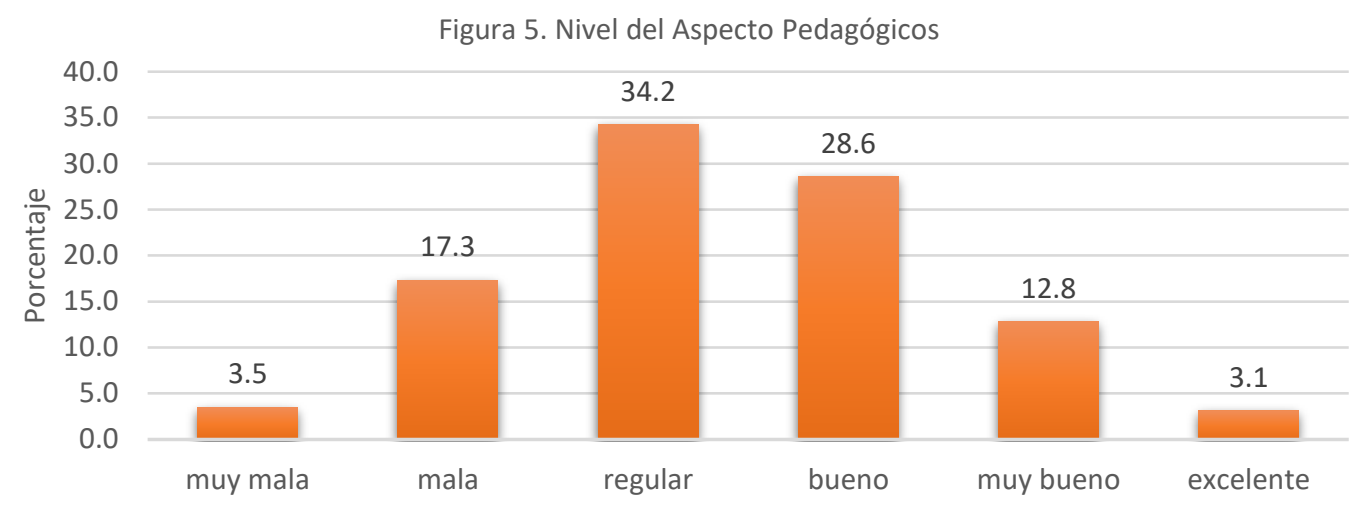

Fuente: Elaboración propia

En la figura 6 se puede ver que en el Aspecto de Gestión y organización escolar el nivel más sobresaliente es el bueno, seguido por el nivel regular y el muy bueno.

Figura 6. Nivel del Aspecto de gestión y organización escolar

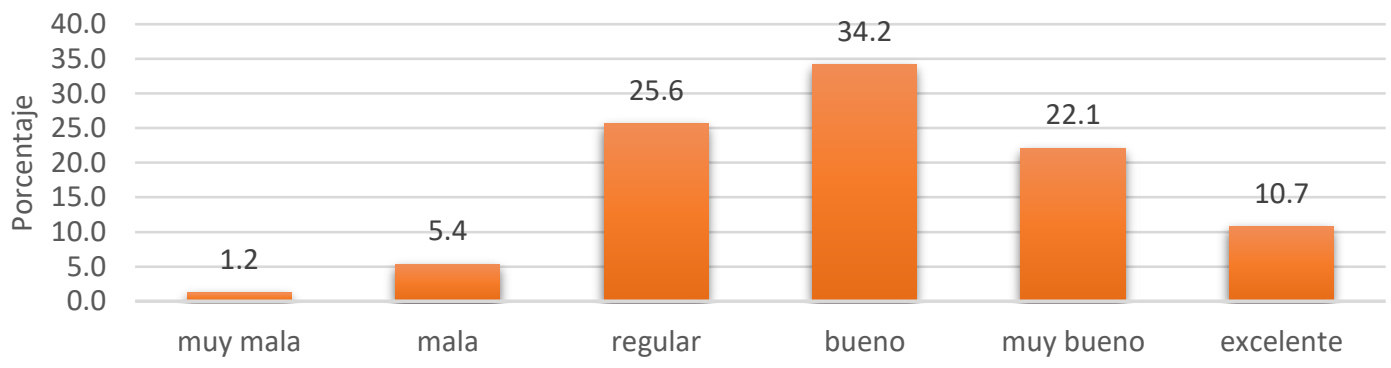

Fuente: Elaboración propia

En el Aspecto de comunicación con nuevas herramientas los adolescentes reflejan un nivel regular, de manera muy cercana se puede ver el nivel bueno, como se informa en la figura 7. 


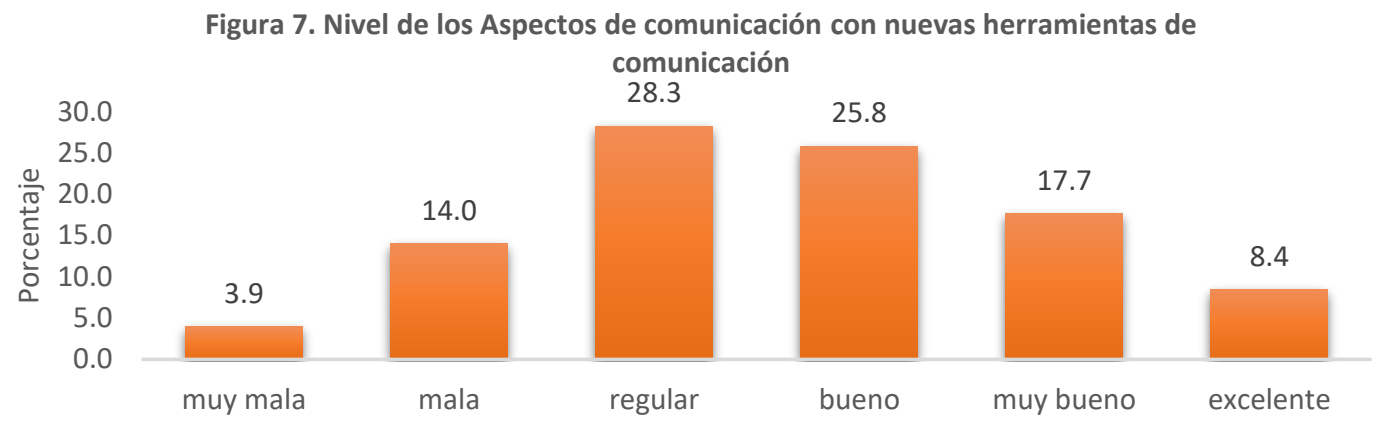

Fuente: Elaboración propia

Como se puede observar en la figura 8 refleja que el nivel bueno es el más alto que los jóvenes reflejan en el Aspecto Social, Ético y Legal y de manera muy cercana los niveles muy bueno y regular.

Figura 8. Aspectos Sociales,éticos y legales

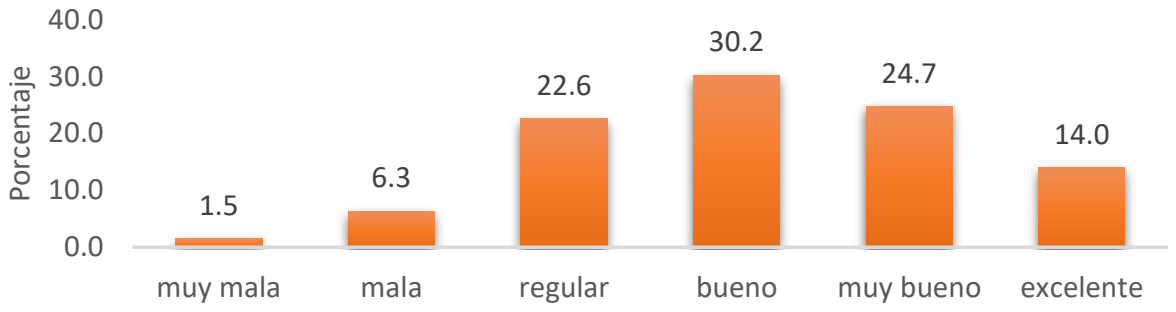

Fuente: Elaboración propia

En lo que respecta de manera global el nivel de las Competencias Tecnológicas que destacan los jóvenes del nivel medio superior es de un $35.6 \%$ como buenas competencias, seguida de un $31.8 \%$ del nivel regular, como se muestra en la figura 9.

Figura 9. Niveles de las Competencias Tecnológicas

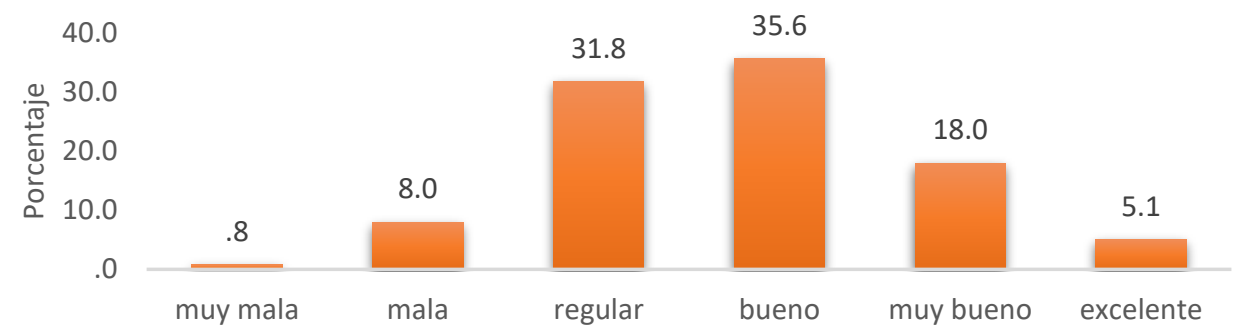

Fuente: Elaboración propia 
Llama la atención que, con respecto a las competencias tecnológicas que se muestra en la figura 10, los hallazgos se puede observar que los adolescentes cuentan con competencias tecnológicas con niveles entre buenos y regulares, en los Aspectos técnicos, pedagógicos, de gestión y organización escolar y aspectos sociales, éticos y legales y un nivel regular en el Aspectos de comunicación con nuevas herramientas de comunicación.

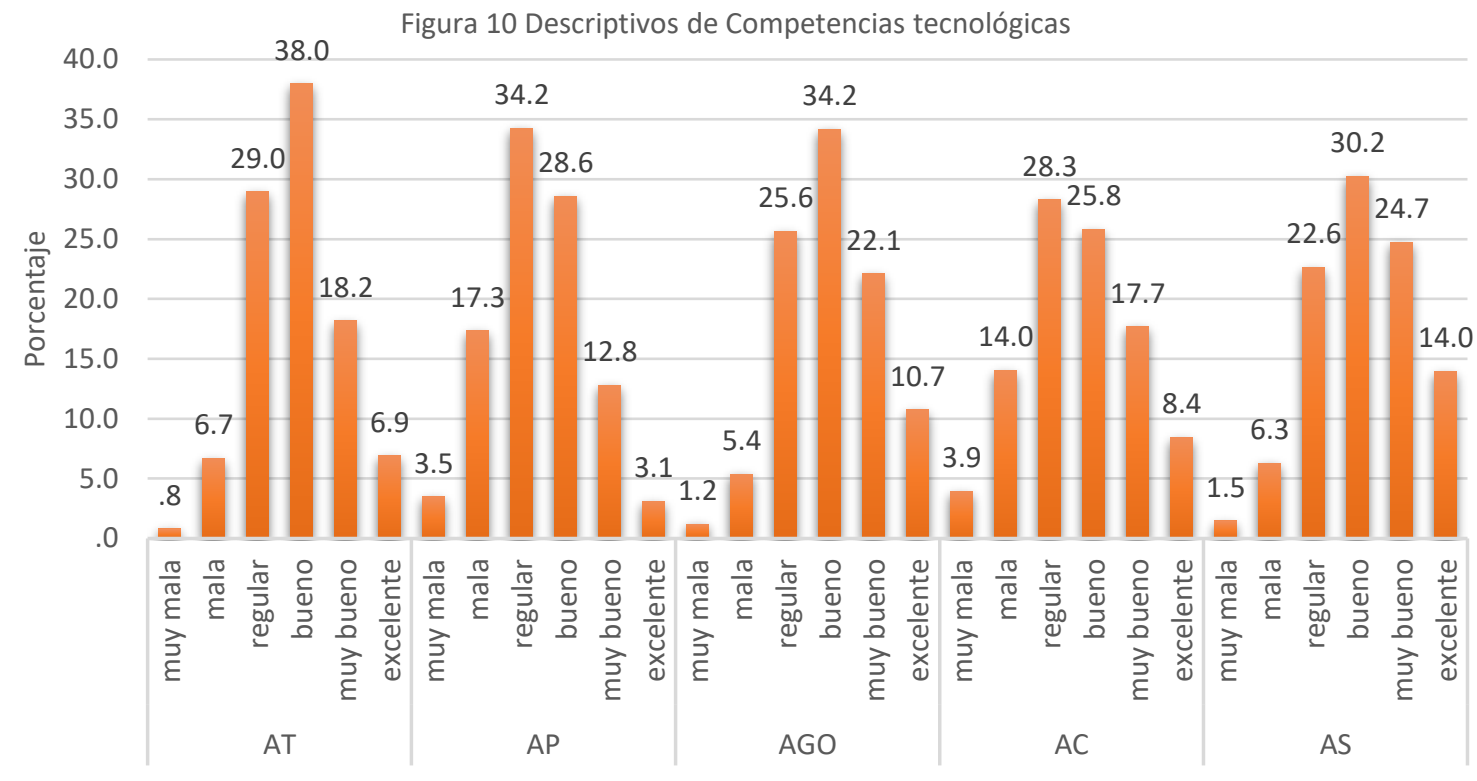

Fuente: Elaboración propia

En torno a la asociación entre las variables que integran el concepto de Competencias tecnológicas, tal y como se muestra en la tabla 3, existen correlaciones positivas entre los distintos aspectos que integran la variable competencia tecnológica.

\begin{tabular}{|c|c|c|c|c|}
\hline & $\mathbf{A P}$ & AGO & $\mathbf{A C}$ & $\mathbf{A S}$ \\
\hline AT & $.726^{* *}$ & $.649^{* *}$ & $.546^{* *}$ & $.770^{* *}$ \\
\hline $\mathbf{A P}$ & & $.658^{* *}$ & $.504^{* *}$ & $.641^{* *}$ \\
\hline AGO & & & $.500^{* *}$ & $.588^{* *}$ \\
\hline $\mathrm{AC}$ & & & & $.503^{* *}$ \\
\hline
\end{tabular}

Nota: ${ }^{* *} \mathrm{p}<.01$. 
En los resultados se puede observar que el $17.2 \%$ de los hombres mostraron un nivel de competencia buena, por otro lado el $18.7 \%$ de las mujeres mostraron ese mismo nivel de competencia. Un $15.1 \%$ de los hombres de la muestra manifestó un nivel regular en la variable competencias. Mientras que el $16.9 \%$ de las mujeres mostró un nivel regular de competencias $\left(\mathrm{chi}^{2}=.618, \mathrm{gl} .=5, \mathrm{Sig}=.987\right)$.

Y los últimos análisis para saber si existe independencia entre las variables género y Aspectos de gestión y organización escolar, se puede observar que el $16.9 \%$ de los hombres mostraron un nivel de bueno, por otro lado el $17.5 \%$ de las mujeres mostraron un nivel bueno en lo que respecta a esta variable. En este sentido el $12.9 \%$ de los hombres de la muestra manifestó un nivel regular mientras que el 12.9\% de las mujeres mostró un nivel regular de la variable Aspectos de gestión y organización escolar la cual se refiere a la confiabilidad que le da el profesor a la información encontrada $\left(\mathrm{chi}^{2}=10.674\right.$, gl. $=5$, Sig= $.058)$.

\section{CONCLUSIONES}

Los hallazgos destacan que en el Aspecto técnico, el cual evalúa si el sujeto tiene Conocimientos básicos sobre el funcionamiento de la computadora y sus periféricos, además de las funciones básicas de ésta se puede ver que los niveles de los estudiantes van de desde bueno, regular y muy bueno, mostrando con ello que los nativos digitales cuentan con conocimientos básicos en este sentido. Por otra parte se puede ver que en el Aspecto Pedagógico el cual se refiere, al empleo que los alumnos hacen de las TIC en su proceso de aprendizaje, presentan niveles de regular, bueno y malo, lo cual es un aspecto a mejorar y trabajar, ya que los alumnos conocen su uso, pero no de qué forma pueden utilizar las Tecnologías de Información y Comunicación como una herramienta de apoyo en su proceso de aprendizaje, con la finalidad de desarrollar como lo menciona Monereo (2003) una competencia para buscar información y aprender a aprender.

Posteriormente en el Aspecto de Gestión y organización escolar que valora el uso de la computadora e internet para almacenar, organizar y tratar documentación sobre informaciones solicitadas utilizadas en los procesos de enseñanza y aprendizaje, los jóvenes revelan en porcentajes altos, tener niveles como bueno, regular y muy bueno, lo que indica que saben aprovechar todos los servicios que la computadora e Internet proporciona. De 
igual manera en el Aspecto de Comunicación con nuevas herramientas que determina la utilización de las TIC como medio de contacto con el profesor y con sus pares, evidencia que los estudiantes cuentan con niveles como regular, bueno y muy bueno, lo cual nos sigue demostrando que saben utilizar las TIC, nos confirma el desarrollo de la competencia que define Monereo (2013) como Competencia para aprender a comunicarse y la que categoriza Llorente y Cerro (2005) como Herramientas tecnológicas para la comunicación.

De igual manera en el Aspecto Social, Ético y legal que se refiere a la confiabilidad, que le da el estudiante a la información encontrada con el empleo de las TIC, se prueba que cuentan con niveles bueno, muy bueno y regular, indicando que pueden priorizar entre la información fidedigna y falsa que podemos encontrar en el Internet, lo que Llorente y Cerro (2005) refieren como Herramientas tecnológicas para la investigación.

En conclusión, los resultados anteriores nos llevan a realizar una propuesta donde se implementen y desarrollen las competencias básicas (como aprender a hacer, aprender a ser y aprender a saber) y posteriormente las competencias TIC (Herramientas tecnológicas para la productividad, Herramientas tecnológicas para la comunicación e investigación y herramientas para la solución de problemas y toma de decisiones ), con el fin de que los estudiantes tengan educación con calidad, un aprendizaje y conocimiento óptimo para el mundo que les aguarda al concluir su proceso formativo.(Llorente y Cerro,2005). 


\section{Bibliografía}

Acuña, A. (2012).Competencias tecnológicas en los alumnos de secundaria y bachillerato. El uso de las Tecnologías de Información y la Comunicación (TIC) en la Educación. ANUIES.

Blanco, A. (Ed.). (2009). Desarrollo y Evaluación de Competencias en Educación Superior. Editorial:Narcea

Guadarrama, H. (2012). Competencias docentes. México: ESFINGE

Guerra, M.A, Moreno, I. y Marquina, R. (2008). Nativos e Inmigrantes Digitales. Recuperado el 15 de octubre del 2016. Consultado en: http://www.protecciononline.com/\%C2\%BFque-es-un-nativo-digital-y-unmigrante-digital/

Llorente, C, y Cerro, S. (2005). Competencias tecnológicas en los alumnos de secundaria y bachillerato. En V Congreso Internacional Virtual de Educación. Recuperado de: cibereduca.com. Recuperado el 30 de julio del 2016. Consultado en: http://tecnologiaedu.us.es/cuestionario/bibliovir/llorente.pdf

Llorente,M.C, y Cabero, J. (2009). Desarrollo de un instrumento sobre competencias tic en alumnos universitarios. Recuperado el 30 de julio del 2016. Consultado en: http://www.gabinetecomunicacionyeducacion.com/sites/default/files/field/adjuntos/ desarrollo_de_un_instrumento_sobre_competencias_tic_en_alumnos_universitarios. pdf

Monereo, C. (2003). Internet, un espacio idóneo para desarrollar las competencias básicas. Aulas de innovación. Recuperado el 15 de octubre del 2016. Consultado en:https://www.researchgate.net/profile/Carles_Monereo/publication/260815787_In ternet_un_espacio_idoneo_para_desarrollar_las_competencias_basicas/links/00b49 5326d4909bcd5000000.pdf?inViewer=0\&pdfJsDownload=0\&origin=publication_d etail. Editorial: GRAO

Pimienta, J.H. (2012). Las competencias en la Docencia Universitaria. México: Pearson Educación. 


\section{AGRADECIMIENTOS}

PROYECTO REALIZADO CON EL FINANCIAMIENTO PARCIAL DE LA SECRETARIA DE EDUCACIÓN PÚBLICA-SUBSECRETARIA DE EDUCACIÓN SUPERIOR-DIRECCIÓN GENERAL DE EDUCACIÓN SUPERIOR UNIVERSITARIA, PADES; PROYECTO No. 2016-16-001-018 